\title{
A Theorem of Sarkovskii on the Existence of Periodic Orbits of Continuous Endomorphisms of the Real Line
}

\author{
P.Stefan
}

Institut des Hautes Etudes Scientifiques, F-91440 Bures-sur-Yvette, France

\begin{abstract}
Two theorems are proved - the first and the more important of them due to Sarkovskii-providing complete and surprisingly simple answers to the following two questions: (i) given that a continuous map $T$ of an interval into itself (more generally, into the real line) has a periodic orbit of period $n$, which other integers must occur as periods of the periodic orbits of $T$ ? (ii) given that $n$ is the least odd integer which occurs as a period of a periodic orbit of $T$, what is the "shape" of that orbit relative to its natural ordering as a finite subset of the real line? As an application, we obtain improved lower bounds for the topological entropy of $T$.
\end{abstract}

Consider an order relation $\vdash$ on the set $N$ of all integers $\geqq 1$, defined as follows. Let $N=A \cup B, A=\left\{2^{n} l: n \leqq 0, l \leqq 3, l\right.$ odd $\}$, and $B=\left\{2^{m}: m \leqq 0\right\}$. Order $A$ lexicographically with increasing $n$ and $l$; order $B$ with decreasing $m$, and let $A$ precede $B$. We have

$$
3 \vdash 5 \vdash 7 \vdash 9 \vdash \ldots \vdash 2 \cdot 3 \vdash 2 \cdot 5 \vdash \ldots \vdash 4 \cdot 3 \vdash \ldots \vdash 8 \vdash 4 \vdash 2 \vdash 1 \text {. }
$$

The main result of [1] is

Theorem 1 (Šarkovskii). Let $T: \mathbb{R} \rightarrow \mathbb{R}$ be a continuous mapping which has a periodic orbit of period $n$. Then $T$ has a periodic orbit of period $m$ for every $m \in N$ such that $n \vdash m$.

The main aim of these notes is to make the contents of [1] available to those who do not read Russian. The reader should be warned that this is not a translation: some new results, closely related to Šarkovskii's work, are presented in Sections $E$ and $H$, and the material of [1] has been rearranged and modified to suit my taste and to avoid one or two mistakes which have crept into Šarkovskii's argument. Nonetheless, I believe that all the main points of [1] and here, and I have tried not to omit anything potentially useful.

The proof of Theorem 1 occupies Sections A-D below. Section E contains the proof of the fact that the "minimal" odd orbits are, up to an order preserving or order reversing isomorphism, uniquely determined by their period. This result 
(whose proof depends heavily on Šarkovskii’s ideas) is not taken from [1] and is probably new. The construction of continuous maps which have a prescribed set of periods is described in Section F. Section G contains some further comments and outlines the differences between these notes, [1], and the related portions of [3] and [4]. Finally, as an application of Šarkovskii's theorem and our result on minimal orbits, we show in Section $\mathrm{H}$ how to strengthen the recently obtained [2] lower bounds for the topological entropy and the number of periodic points of $T$, simplifying at the same time the proofs of these estimates.

I should like to thank Sheldon Newhouse for bringing Šarkovskii's work to my attention and for several very helpful discussions, and Joel Lebowitz for his interest and encouragement during the preparation of these notes.

\section{A. Definitions and Remarks}

If $T: X \rightarrow X$ is a mapping of a set $X$ into itself, the orbit of $x \in X$ relative to $T$ is the set $\omega(T, x)=\left\{x, T x, T^{2} x, \ldots\right\}$. An orbit $\omega$ of $T$ is periodic if it is finite and if $T$ restricts to a bijective map $\omega \rightarrow \omega$; the period of $\omega$ is its cardinality \# $\omega$. We write $\operatorname{Per}(T, k)$ for the union of all periodic orbits of $T$ with period $k$, and Fix $T=\operatorname{Per}(T, 1)$ for the set of fixed points of $T$. Clearly

(1) $x \in \operatorname{Fix}\left(T^{k}\right)$ iff $\omega(T, x)$ is periodic and its period divides $k$;

(2) $\operatorname{Per}(T, n) \subset \operatorname{Per}\left(T^{m}, n /\right.$ h.c.f. $\left.(n, m)\right)$;

(3) If $p$ is prime, then $\operatorname{Per}\left(T, p^{n+m}\right)=\operatorname{Per}\left(T^{p^{n}}, p^{m}\right)$.

From now on we assume that $X=\mathbb{R}$ and that $T$ is continuous.

We write $\mathbb{R}=\operatorname{Fix}(T) \cup U \cup D$, where $U=U(T)$ is the open set $\{x \in \mathbb{R}: T x>x\}$ and $D=D(T)=\{x \in \mathbb{R}: T x<x\}$. If $\omega$ is a periodic orbit of $T$, we write $\omega_{\min }=\min \omega, \omega_{\max }$ $=\max \omega, \omega^{U}=\max (\omega \cap U)$, and $\omega^{D}=\min (\omega \cap D)$ (see Fig. 1). If Fix $T=\emptyset$ then clearly either $\mathbb{R}=U$ or $\mathbb{R}=D$, and so

(4) If $T$ has a periodic orbit, then it has a fixed point.

Fig. 1

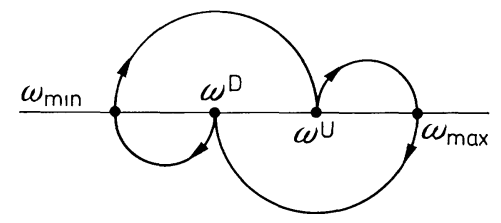

\section{B. Existence of Arbitrary Periods}

(5) Let $L$ and $R$ be two closed intervals such that $\max L=\lambda \leqq \varrho=\min R$. If (i) $L \cup R \subset T R$ and $R \subset T L$, and (ii) $\lambda<\varrho$ or $T^{2} \lambda \notin R$, then $\operatorname{Per}(T, n) \neq \emptyset$ for all $n \geqq 1$.

Proof (Li and Yorke). Put $I_{k}=L$ if $k \equiv(n-1) \bmod n, I_{k}=R$ otherwise. Since $I_{k+1} \subset T\left(I_{k}\right)$, we can find a sequence $\left(A_{k}\right)$ of closed intervals such that $A_{0}=I_{0}=R$, $A_{k+1} \subset A_{k}$, and $T^{k} A_{k}=I_{k}$. As $T^{n} A_{n}=I_{n}=R \supset A_{n}, T^{n}$ has a fixed point $a \in A_{n}$. We have $\left\{a, T a, \ldots, T^{n-2} a\right\} \subset R$ and $T^{n-1} a \in L$.

If $T^{n-1} a \in R$, then $T^{n-1} a=\lambda=\varrho$ and $T^{2} \lambda=T a \in R$, an impossibility. Hence $\# \omega(T, a)=n$. 
(6) If $T^{3} a \leqq a<T a<T^{2} a$ (or $T^{3} a \geqq a>T a>T^{2} a$ ) for some $a \in \mathbb{R}$, then $\operatorname{Per}(T, n) \neq \emptyset$ for all $n \geqq 1[4]$.

Proof. Take $L=[a, T a], R=\left[T a, T^{2} a\right]$ and use (5).

In particular

(7) If $\operatorname{Per}(T, 3) \neq \emptyset$, then $\operatorname{Per}(T, n) \neq \emptyset$ for all $n \geqq 1$.

(8) If $T b<c<a<b \leqq T a$ (or $T b>c>a>b \geqq T a$ ) and $T c=c$, then $\operatorname{Per}(T, n) \neq \emptyset$ for all $n \geqq 1$.

Proof. Choose $d \in[c, a]$ such that $T d=b$ and put $L=[c, d], R=[d, b]$.

(9) Let $\omega$ be a periodic orbit of $T$. If $T$ has a fixed point between $\omega_{\min }$ and $\omega^{U}$ (or between $\omega^{D}$ and $\left.\omega_{\max }\right)$, then Per $(T, n) \neq \emptyset$ for all $n \geqq 1$.

Proof. Let $\omega_{\min }<c<\omega^{U}, T c=c, A=\{x \in \omega \cap U: x>c\}$. Choose $a \in A$ such that $T a=\max \{T x: x \in A\}$. Let $d \leqq c$ be the greatest fixed point of $T$ in $[c, a]$. Since $\omega_{\min }<c \leqq d$, the sequence $a, T a, T^{2} a, \ldots$ cannot remain forever in $[d, T a]$. But if $x \in \omega \cap[d, T a]$, then either $x \in A$ and $T x \leqq T a$, or $x \in D$ and $T x<x \leqq T a$. Hence there exists $b \in[d, T a]$ such that $T b<d$. Since $T a>a, b<a$ would force a fixed point of $T$ between $b$ and $a$, contrary to the definition of $d$. Hence $T b<d<a<b \leqq T a$, and we may use (8).

(10) Let $\omega$ be a periodic orbit of $T$. If $\omega^{D}<\omega^{U}$, then, by (9), $\operatorname{Per}(T, n) \neq \emptyset$ for all $n \geqq 1$.

For example, if $\omega$ is as in Figure 1, then $T$ has periodic orbits of arbitrary periods.

(11) If $\operatorname{Per}(T, n) \neq \emptyset$ for some odd $n \geqq 3$, then $\operatorname{Per}\left(T^{2}, m\right) \neq \emptyset$ for all $m \geqq 1$.

Proof. Let $\omega$ be a periodic orbit of $T$ with period $n$; since $n$ is odd, $\omega$ is also a periodic orbit of $T^{2}$. Let

$$
\omega_{2}^{U}=\max \left(\omega \cap U\left(T^{2}\right)\right), \quad \omega_{2}^{D}=\min \left(\omega \cap D\left(T^{2}\right)\right) .
$$

By (10), we may assume that $\omega_{2}^{U}<\omega_{2}^{D}$. Similarly, if $\omega^{D}<\omega^{U}$, then $T$ has arbitrary periods by (10) and so $T^{2}$ has arbitrary periods by (2); we may therefore also assume that $\omega^{U}<\omega^{D}$. Note that then $\omega$ has no elements between $\omega^{U}$ and $\omega^{D}$ and between $\omega_{2}^{U}$ and $\omega_{2}^{D}$. There are therefore three cases consider : $\omega_{\min } \leqq \omega_{2}^{U}<\omega_{2}^{D} \leqq \omega^{U}<\omega^{D} \leqq \omega_{\max }$, $\omega_{\min } \leqq \omega^{U}<\omega^{D} \leqq \omega_{2}^{U}<\omega_{2}^{D} \leqq \omega_{\max }$, and $\omega_{\min } \leqq \omega^{U}=\omega_{2}^{U}<\omega_{2}^{D}=\omega^{D} \leqq \omega_{\max }$. But $T$ (and hence $T^{2}$ ) has a fixed point $\gamma$ between $\omega^{U}$ and $\omega^{D}$, and so in the first two cases $T^{2}$ has arbitrary periods by (9). It remains to consider the third case. Let $a \in \omega, T a=\omega_{\min }$. Then $a>T a$ and so $a>\omega^{D}$. Let $b \in \omega, T b=a$ : then $b>T^{2} b=\omega_{\min }$, and so $b>\omega_{2}^{D}=\omega^{D}$. But then $b>T b=a \geqq \omega^{D}$. Let $c \in \omega, \quad T c=\omega_{\max }$. Then $T^{2} b=\omega_{\min } \leqq c \leqq \omega^{U}<T b$. Hence there exists $d \in \mathbb{R}$ such that $T b \leqq d<b$ and $T d=c$. We have $T^{2} b<\gamma<d<b \leqq T^{2} d$, and so $T^{2}$ has arbitrary periods by (8).

\section{Proof of the Main Theorem}

(12) If $T$ has a periodic orbit of period $\geqq 2$, then it has a periodic orbit of period 2 .

Proof. We assume that $\operatorname{Per}(T, 2)=\emptyset$ and prove by induction that $\operatorname{Per}(T, n)=\emptyset$ for all $n \geqq 2$ : Let $\operatorname{Per}(T, 2)=\ldots=\operatorname{Per}(T, n)=\emptyset$, and let $a \in \operatorname{Per}(T, n+1)$. Let $C_{k}$ be the connected component of $T^{k} a$ in $\mathbb{R} \backslash F i x T$. As $\operatorname{Fix}(T)=\operatorname{Fix}\left(T^{2}\right)=\ldots=\operatorname{Fix}\left(T^{n}\right)$, the sign of $T^{p} x-x$ is constant on each $C_{k}$ for $p \leqq n$. We claim that, for $x \in \cup C_{k}$ and $1 \leqq p \leqq n$, 
For if $C_{k}$ is between two fixed points, then $C_{k} \subset T^{m} C_{k}$ for all $m \geqq 1$, and so there exists $y$ such that $\left\{y, T y, \ldots, T^{n} y\right\} \subset C_{k}$. If $C_{k}$ is (say) on the left of all fixed points of $T$, we have $T^{p} x>x$ on $C_{k}$ : else $x>T^{p} x>T^{2 p} x>\ldots$ and $C_{k}$ could not meet a periodic orbit. This proves (*). If now say $a<T a$, then $a<T^{2} a$. As $T^{n}(T a)=a<T a$, we have $a<T^{2} a<T a$. But now $T^{n-1}\left(T^{2} a\right)=T^{n+1} a=a<T^{2} a, T^{n}\left(T^{2} a\right)=T a>T^{2} a$, a contradiction.

(13) If $\operatorname{Per}\left(T, 2^{n}\right) \neq \emptyset$, then $\operatorname{Per}\left(T, 2^{k}\right) \neq \emptyset$ for $0 \leqq k \leqq n$.

Proof. Let $m=2^{k-1}$. By (2), $\operatorname{Per}\left(T^{m}, 2^{n-k+1}\right) \# \emptyset$. Now use (12) and (3).

(14) If $\operatorname{Per}\left(T, 2^{k} l\right) \neq \emptyset$ for some odd $l \geqq 3$ and some $k \geqq 0$, then $\operatorname{Per}\left(T, 2^{n}\right) \neq \emptyset$ for all $n \geqq 0$.

Proof. Use (2), (12), and (13).

(15) Lemma. Let $\operatorname{Per}(T, n) \neq \emptyset$ for some $n \geqq 3, n$ odd.

(a) If $k \geqq 2 n$, then $\operatorname{Per}(T, k) \neq \emptyset$.

(b) If $k>n$, then $T^{k}$ has a fixed point $\varrho$ such that $\# \omega(T, \varrho) \geqq 3$.

The proof is given in Section D.

(16) Let $\dot{\operatorname{Per}}(T, n) \neq \emptyset$ for some odd $n \geqq 3$. Then $\operatorname{Per}(T, k) \neq \emptyset$ for all odd $k \geqq n$.

Proof. Let $\varrho$ be as in (15b) and let $r=\# \omega(T, \varrho)$. Then $r \geqq 3, r$ divides $k$, and $r$ is odd. If $r=k$, we are done. Otherwise, $r \leqq k / 3$. By $(15 . \mathrm{a}), \operatorname{Per}(T, m) \neq \emptyset$ for all $m \geqq 2 r$ and so $\operatorname{Per}(T, k) \neq \emptyset$.

(17) If $\operatorname{Per}\left(T^{2}, n\right) \neq \emptyset$ for some $n \geqq 2$, then $\operatorname{Per}(T, 2 n) \neq \emptyset$.

Proof. Let $a \in \operatorname{Per}\left(T^{2}, n\right), r=\# \omega(T, a)$. Clearly $r \geqq n$ and $r$ divides $2 n$. If $n$ is even, then $T^{n} a \neq a$ and $r=2 n$ : we may assume that $r=n$ and that $n$ is odd. But then $n \geqq 3$ and the result follows from (15.a).

(18) If $\operatorname{Per}(T, n) \neq \emptyset$ for some odd $n \geqq 3$, then $\operatorname{Per}(T, 2 k) \neq \emptyset$ for all $k$.

Proof. This follows at once from (11) and (17).

(19) Let $\operatorname{Per}\left(T, 2^{n} l\right) \neq \emptyset$ for some $n \geqq 0, l \geqq 3, l$ odd. Then $\operatorname{Per}\left(T, 2^{m} k\right) \neq \emptyset$ whenever $m>n$ or $m=n$ and $k \geqq l$.

Proof. For $n=0$, this is just (16) and (18). Assume that (19) has been proved for $n-1$. $\operatorname{By}(2), \operatorname{Per}\left(T^{2}, 2^{n-1} l\right) \neq \emptyset$. By induction hypothesis, $\operatorname{Per}\left(T^{2}, 2^{m-1} k\right) \neq \emptyset$ for $m>n$ and $m=n, k \geqq l$. Now use (17).

Theorem 1 now follows at once from (13), (14), and (19).

\section{Proof of Lemma 15. Remarks}

Let $\omega$ be a periodic orbit of $T$, of odd period $n \geqq 3$. By (10), we may assume

(a) $\omega^{U}<\omega^{D}$.

As $n$ is odd, there exists $a$ such that either

(b) $\{a, T a\} \subset \omega \cap U$ 
or $\{a, T a\} \subset \omega \cap D$; without loss of generality we may assume (b). The rest of the argument applies to any periodic orbit satisfying (a) and (b); the fact that $n$ is odd is not used again.

We claim that there exists $b$ and $c$ in $\omega$ and an integer $m$ such that

(c) $T^{m} c \leqq a<T a \leqq b<c \leqq T b$ and $T c<T a$;

(d) $1 \leqq m \leqq n-2$.

To show this, let $q \geqq 2$ be the smallest positive integers such that

(e) $T^{a} b \leqq a<T a \leqq b<T b$

for some $b \in \omega$. Taking $b=T a$, we see that $2 \leqq q \leqq n-1$. As $T^{q-1} T b \leqq a$, we cannot have $T b<T^{2} b$ by the minimality of $q$. Hence $T b \in D$. Let $l \geqq 2$ be the first positive integer for which $T^{l} b \in U$. Thus $\left\{T b, \ldots, T^{-1} b\right\} \subset \omega \cap D$. Since $b \in \omega \cap U$, (a) implies that $b<T^{l-1} b \leqq T b$. Put $c=T^{l-1} b$ and note that $T c \in \omega \cap U$ and so $T c<T^{2} c$. But $T^{q-l} T c=T^{q} b \leqq a$ and so $T c<T a$ by the minimality of $q$. Now put $m=q-l+1$.

Next, we assume (c) and put $p=m+3$. There exists $\xi \in \mathbb{R}$ such that

(f) $\quad T^{p} \xi \leqq a<T a \leqq T^{2} \xi<\xi<T \xi<T^{3} \xi, \quad 4 \leqq p \leqq n+1$;

(g) $T x>T^{2} \xi$ for $T^{2} \xi \leqq x<T \xi$.

(Choose $\eta$ in $[b, c]$ such that $T \eta=c$ and the smallest $\zeta$ in $[\eta, c]$ such that $T \zeta=\eta$; note that $T x>\eta$ if $\eta \leqq x<\zeta$. Now choose $\xi$ in $[\eta, \zeta]$ such that $T \xi=\zeta$.)

Observe that $T^{2}$ maps $[\xi, T \xi]$ onto a larger interval, and so $T^{2}$ must have fixed points between $\xi$ and $T \xi$ : let $\omega_{1}$ and $\omega_{2}$ be the smallest and the largest of these. We have

(h) $T^{p} \xi \leqq a<T a \leqq T^{2} \xi<\xi<\omega_{1} \leqq \omega_{2}<T \xi<T^{3} \xi$;

(i) $\left[\xi, \omega_{1}\left[\subset D\left(T^{2}\right),\right] \omega_{2}, T \xi\right] \subset U\left(T^{2}\right)$.

We find sequences $\left(\alpha_{i}\right)$ and $\left(\beta_{i}\right)$ such that

(j) $\xi=\alpha_{0}<\alpha_{1}<\ldots<\alpha_{i}<\ldots<\omega_{1} \leqq \omega_{2}<\ldots<\beta_{i}<\ldots<\beta_{1}<\beta_{0}=T \xi$;

(k) $\quad T^{2} \alpha_{i}=\alpha_{i-1}, T^{2} \beta_{i}=\beta_{i-1}$;

(l) $\left.T^{2}\right] \alpha_{i}, \omega_{1}[=] \alpha_{i-1}, \omega_{1}\left[, T^{2}\right] \omega_{2}, \beta_{i}[=] \omega_{2}, \beta_{i-1}[, i \geqq 1$.

Next, find $\lambda_{1}$ and $\lambda_{2}$ between $a$ and $T^{2} \xi$ such that

(m) $T \lambda_{1}=\omega_{1}$ and $T x<\omega_{1}$ for $a \leqq x<\lambda_{1}$;

(n) $T \lambda_{2}=\omega_{2}$ and $T x>\omega_{2}$ for $\lambda_{2}<x \leqq T^{2} \xi$.

Finally, we find sequences $\left(\sigma_{i}\right)$ and $\left(\tau_{i}\right)$ such that

(o) $a \leqq \sigma_{-1}<\sigma_{0}<\ldots<\sigma_{i}<\ldots<\lambda_{1} \leqq \lambda_{2}<\ldots<\tau_{i}<\ldots<\tau_{0}<\tau_{-1} \leqq T^{2} \xi$;

(p) $T \sigma_{i}=\alpha_{i}$ and $T \tau_{i}=\beta_{i}$ for $i \geqq-1$;

(q) $T] \sigma_{i}, \lambda_{1}[=] \alpha_{i}, \omega_{1}[, T] \lambda_{2}, \tau_{i}[=] \omega_{2}, \beta_{i}[$ for $i \geqq-1$, where we take $\alpha_{-1}=T^{2} \xi$ and $\beta_{-1}=T^{3} \xi$. 
We are now in a position to prove (15).

Let first $k=p+2 i, i \geqq 0$. We have $T^{k} \lambda_{2}=T^{k-1} \omega_{2}>\lambda_{2}$ by $(\mathrm{g})$ and because $\omega_{2}$ is a fixed point of $T^{2} ; T^{k} \tau_{i}=T^{p-1} T^{2 i} \beta_{i}=T^{p-1} T \xi<\tau_{i}$. Hence $T^{k}$ has a fixed point $\varrho$

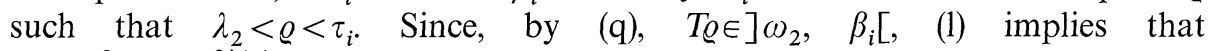
$\left.\left.\left\{T \varrho, T^{3} \varrho, \ldots, T^{2 i+1} \varrho\right\} \subset\right] \omega_{2}, T \xi\right]$.

Hence, by (i),

(r) $\varrho<T^{2} \xi<\omega_{2}<T \varrho<T^{3} \varrho<\ldots<T^{2 i+1} \varrho<T^{2 i+3} \varrho$.

Let $r=\# \omega(T, \varrho)$. If $r \leqq 2 i+3$, then $r$ must be even and $\leqq 2 i+2$, contradicting $T^{r+1} \varrho>T \varrho$. Hence

(s) $\quad r=\# \omega(T, \varrho) \geqq(k-p)+4 \geqq(k-n)+3$.

If $k=p+2 i+1, i \geqq 0$, we find as before that $T^{k}$ has a fixed point $\varrho$ such that $\sigma_{i}<\varrho<\lambda_{1}$, and therefore

(t) $\varrho<T^{2} \xi<\xi<T^{2 i+1} \varrho<T^{2 i-1} \varrho<\ldots<T \varrho$.

By (g), we also have $T^{2 i+2} \varrho>T^{2} \xi$. As above, we conclude that

(u) $r=\# \omega(T, \varrho) \geqq(k-p)+2 \geqq(k-n)+1$.

If $k \geqq n+2$ or if $k=n+1$ and $p \leqq n$, then $r \geqq 3$ by (s) or (u). But if $k=p=n+1$, then (s) applies and again $r \geqq 3$.

Finally, if $k \geqq 2 n$, then $r>(k-n) \geqq k / 2$ and so, since $r$ divides $k, r=k$. This concludes the proof of (15).

Remark 1. Repeating the above argument for $i=-1$ we obtain $\varrho_{1}$ and $\varrho_{2}$ such that $T^{p-1} \varrho_{1}=\varrho_{1}, T^{p-2} \varrho_{2}=\varrho_{2}, T \varrho_{1} \neq \varrho_{1}$ and $T \varrho_{2} \neq \varrho_{2}$.

Remark 2. Sarkovskii shows that if $p$ is even, $k=p+2 i+1, i \geqq 0$, and $\varrho$ is the greatest fixed point of $T^{k}$ in $\left[\sigma_{i}, \lambda_{1}\right]$, then \# $\omega(T, \varrho)=k$. For assume that $r<k$. Since $k$ is odd and $r$ divides $k, r$ is odd and $k-r=2 q$. By (1), $\alpha_{i}<T \varrho<\omega_{1}$ implies that $T \varrho=T^{2 q} \gamma$ for some $\gamma$ such that $\alpha_{i+q}<\gamma<\omega_{1}$. By (l) and (i), $\gamma>T^{2 q} \gamma=T \varrho$. Hence there exists $\delta$ such that $\varrho<\delta<\lambda_{1}$ and $T \delta=\gamma$. But then $T^{k} \delta=T^{r-1} T^{k-r} T \delta=T^{r} \varrho=\varrho<\delta$. As $T^{k} \lambda_{1}>\lambda_{1}$, this would force a fixed point of $T^{k}$ between $\delta$ and $\lambda_{1}$, contradicting our choice of $\varrho$.

The results of this section are summed up in the next two lemmas.

(20) Lemma. Let $m=m(T)$ be the smallest positive integer ${ }^{1}$ such that $T^{m} c \leqq a<T a \leqq b<c \leqq T b$ and $T c<T a$ for some $a, b$, and $c$ in $\mathbb{R}$. Let $p=p(T)$ be the smallest positive integer ${ }^{1}$ such that $T^{p} \xi \leqq a<T a \leqq T^{2} \xi<\xi<T \xi<T^{3} \xi$ and $T x>T^{2} \xi$ for every $x$ in $\left[T^{2} \xi, T \xi[\right.$, for some $a \in \mathbb{R}$ and $\xi \in \mathbb{R}$. Then (1) $p \leqq m+3 ;$ (2) If $k \geqq p-2$, then there exists $\varrho \in \mathbb{R}$ such that $T^{k} \varrho=\varrho$ and $T \varrho \neq \varrho$; (3) If $k \geqq 2(p-1)$ or if $p$ is even and $k>p$ is odd, then $\operatorname{Per}(T, k) \neq \emptyset$.

(21) Lemma. Let $\omega$ be a periodic orbit of $T$ with (not necessarily odd) period $n \geqq 3$. Assume that $\omega^{U}<\omega^{D}$ and that there exists a $a$ such that $a<T a<T^{2} a$. Let $q=q(\omega)$ be the smallest positive integer such that $T^{q} b \leqq a<T a \leqq b<T b$ for some $a$ and $b$ in $\omega$. If $m=m(T)$ is defined as in (20), then $m+3 \leqq q+2 \leqq n+1$.

1 Or $\infty$, if no such integer exists 


\section{E. Minimal Orbits}

We say that an orbit $\omega$ of $T$ is minimal if (1) $\omega$ is periodic, (2) $n=\# \omega$ is an odd integer $\geqq 3$, and (3) $P(T, m)=\emptyset$ for every odd integer $m$ such that $3 \leqq m \leqq n-2$.

In this section we prove that, for every odd integer $n \geqq 3$, there exists only one "type" of minimal orbit (see Fig. 2). More precisely, note that the restriction of $T$ to a periodic orbit $\omega$ is a cyclic permutation of a finite subset of $\mathbb{R}$. Up to an order preserving isomorphism, there are exactly $(n-1)$ ! such permutations. We shall prove that only two of these $(n-1)$ ! permutations (say $M$ and $P$ ) can possibly be order-isomorphic to a minimal orbit of a continuous mapping $\mathbb{R} \rightarrow \mathbb{R}$. Moreover, $M \cong P$ if we allow order-reversing isomorphisms.

Let $\omega_{1}$ and $\omega_{2}$ be two finite totally ordered sets and let $T_{1}: \omega_{1} \rightarrow \omega_{1}$ and $T_{2}: \omega_{2} \rightarrow \omega_{2}$. We say that $T_{1}$ and $T_{2}$ are isomorphic if there exists a bijective map $\varphi: \omega_{1} \rightarrow \omega_{2}$ such that (1) $\varphi$ is either order preserving or order reversing; and (2) $\varphi \circ T_{1}=T_{2} \circ \varphi$.

Theorem 2. Let $\omega$ be a minimal orbit of a continuous map $T: \mathbb{R} \rightarrow \mathbb{R}, \# \omega=n=2 k+1$. Then $\left.T\right|_{\omega}$ is isomorphic to the permutation $M=M_{n}$ of $\{1,2, \ldots, n\}$, where $M^{2 s+1}(1)$ $=k+1-s$ and $M^{2 s+2}(1)=k+2+s$ for $0 \leqq s \leqq k-1$.

Thus $M_{5}$ is the cyclic permutation $(1,3,4,2,5), M_{7}=(1,4,5,3,6,2,7)$, etc. (see Fig. 2).

Fig. 2

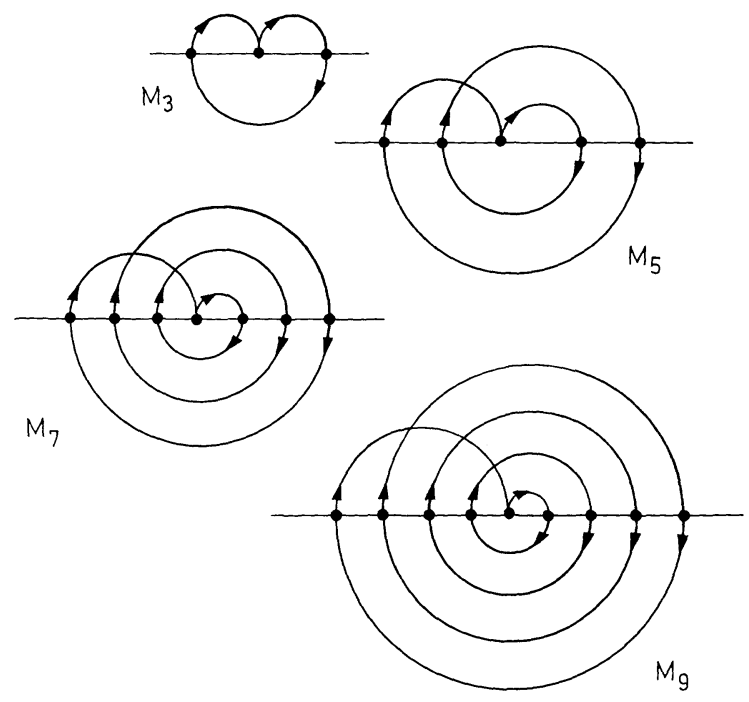

Proof. Let $\omega$ be a minimal orbit of $T$, \# $\omega=n=2 k+1$. Without loss of generality, we may assume that $n \geqq 5$, \# $\omega \cap U>\# \omega \cap D$, and $\omega=\{1,2, \ldots, n\}$. There exists $a \in \omega$ such that

$$
\{a, T a\} \subset \omega \cap U \text {. }
$$

By (10), $\omega^{U}<\omega^{D}$, and so

$$
1=\omega_{\min } \leqq a<T a \leqq \omega^{U}<\omega^{D}=\omega^{U}+1 \leqq \omega_{\max }=n .
$$


Assume first that $a \neq \omega_{\min }$. Then $T^{\mathrm{s}} a=\omega_{\min }$ for some $s, 3 \leqq s \leqq n-1$. Taking $b=T a$ in Lemma (21) we see that, in the notation of that lemma, $q \leqq s-1 \leqq n-2$. But then, by Lemma (20), there exists $\varrho \in \mathbb{R}$ such that $T^{n-2} \varrho=\varrho, T \varrho \neq \varrho$. Since $r=\# \omega(T, \varrho)$ divides $n-2$ which is odd, $r$ is an odd integer $\geqq 3$, contradicting the minimality of $\omega$. This proves

(a) $a=\omega_{\min }=1$;

(b) if $x \in \omega \cap U$ and $x \neq \omega_{\min }$, then $T x \in \omega \cap D$.

It follows that $\# \omega \cap D \geqq \#(\omega \cap U)-1$ and hence $\# \omega \cap D=\#(\omega \cap U)-1$. Hence

(c) $\omega^{U}=k+1$ and $\omega^{D}=k+2$.

Furthermore, since every point of $\omega \cap D$ has pre-image in $\omega \cap U$ and $\left.T\right|_{\omega}$ is injective, the image of every point of $\omega \cap D$ lies in $\omega \cap U$, or

(d) If $x \in \omega$ and $x \geqq k+2$, then $T x \leqq k+1$.

We claim that in fact $T a=\omega^{U}=k+1$. For if not, then $T a<\omega^{U}$. Put $b=\omega^{U}$ in Lemma (21) and let $T^{s} b=a$ for some $s, 1 \leqq s \leqq n$. Since $b \neq a, b \neq T a$, we have in fact $s \leqq n-2$ and therefore $q \leqq n-2$ in Lemma (21). As above, this leads to a contradiction with the minimality of $\omega$ and so

(e) $T a=\omega^{U}=k+1$.

Assume now that $T^{2} a \neq \omega^{D}$. Put $c=\omega^{D}$,

$$
\omega_{\min }=a<T c<T a=\omega^{U}<c<T^{2} a .
$$

Clearly $T^{s} c=a$ for some $s, 2 \leqq s \leqq n-3$. Hence $m \leqq n-3$ in Lemma (20) and we again arrive at a contradiction. Thus

(f) $T^{2} a=\omega^{D}=k+2$.

Summing up what we know and writing $a(s)=T^{s} a$, we obtain

(g) $1=\omega_{\min }=a=a(n)<\ldots<a(3)<\ldots<a(1)<a(2)<\ldots<\omega_{\max }=n=2 k+1$;

(h) $a(1)=k+1$ and $a(2)=k+2$;

(i) $a(2 s+1)<a(1)$ and $a(2 s+2)>a(2)$ for $1 \leqq s \leqq k-1$.

This proves the theorem for $n=5$. For $n \geqq 7$ it is sufficient to show that the finite sequences $(a, a(2), a(4), \ldots)$ and $(a(1), a(3), a(5), \ldots)$ are monotonic. This is proved by induction, starting from $a<a(2)<a(4)$ and $a(1)>a(3)$ and using (i) together with the following result.

(j) If $x<T^{2} x$ and $T^{4} x<T^{2} x \quad$ (or $x>T^{2} x \quad$ and $\quad T^{4} x>T^{2} x$ )

for some $\quad x \in \omega$, then $T^{4}(x) \leqq a(1)<a(2) \leqq T^{2}(x)$

[or $\left.T^{4}(x) \geqq a(2)>a(1) \geqq T^{2}(x)\right]$.

To prove (j), note that $T^{n-2} T^{2} x=x<T^{2} x$ and $T^{n-2} T^{4} x=T^{2} x>T^{4} x$. Hence $T^{n-2}$ has a fixed point $\varrho$ between $T^{2} x$ and $T^{4} x$. By minimality, $\varrho$ must be a fixed point of $T$. But then (9) implies $\omega^{U}<\varrho<\omega^{D}$, proving (j) and concluding the proof of the theorem. 


\section{F. Examples}

(a) Continuous maps $\mathbb{R} \rightarrow \mathbb{R}$ with minimal orbits of a given odd period $n$ are obtained as piece-wise monotonic extensions of the cyclic permutation $M_{n}$ defined in the preceding section (Fig. 3).

Fig. 3

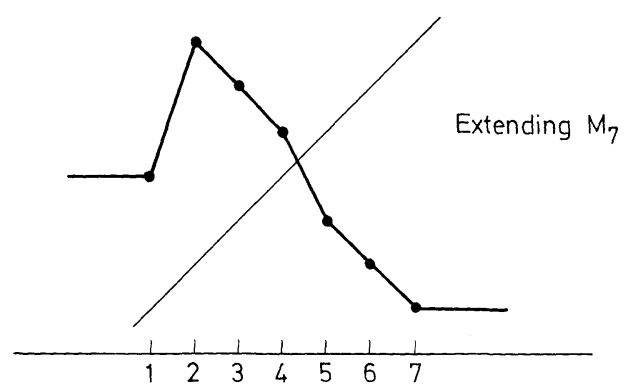

To indicate why these mappings do not have odd periods between 3 and $n-2$, take $n=5$ (the general case is similar). Labelling the intervals as in Figure 4 we note that, under the action of $T$.

(*) $1 \rightarrow 2, \quad 3 \rightarrow 4 \rightarrow 5, \quad 2 \rightarrow 1 \cup 3, \quad$ and $\quad 5 \rightarrow 1 \cup 2 \cup 4$.

Note also that the fixed point $S$ acts as a source: the interval 1 is "stretched" by $T^{2}$ onto $1 \cup 3$. Hence no periodic orbit remains forever in $1 \cup 2$. On the other hand, a periodic orbit which remains in $5 \cup 4$ must be of an even period. Hence periodic orbits with odd period $k \geqq 3$ meet the interval 3 . By $\left(^{*}\right), k \geqq 5$.

Fig. 4

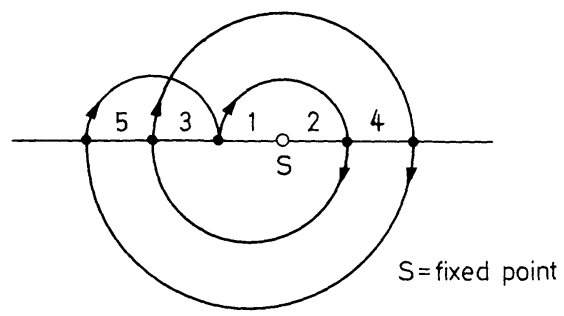

(b) It is easy to give examples of maps with $\operatorname{Per}(T, n)=\emptyset$ for $n \neq 1$ (or $n \neq 1,2)$ and with periodic orbits of period one (or of period 1 and 2).

(c) Other examples are obtained using the "square root" construction: If $T:[a, b] \supset$ and $K>b-a$, we define a "square root" $S$ of $T$ by $S(x)=T(x)+K$ on $[a, b], S(x)=x-K$ on $[a+K, b+K]$ and extend $S$ monotonically across $[b, a+K]$ (see Fig. 5,6). It is clear that, apart from the fixed point in $[b, a+k]$, every periodic orbit $\gamma$ of $S$ meets a unique periodic orbit $\omega$ of $T, \# \gamma=2 \# \omega$.

(d) Starting from (a) or (b) and iterating the construction (c) we obtain mappings with $\operatorname{Per}(T, n) \neq \emptyset$ for a given $n$ and $\operatorname{Per}(T, k)=\emptyset$ for all $k \vdash n$ (see p. 237). With a little care we can also construct continuous maps $T: \mathbb{R} \rightarrow \mathbb{R}$ such that $\operatorname{Per}(T, n) \neq \emptyset$ if and only if $n$ is a power of 2 . 


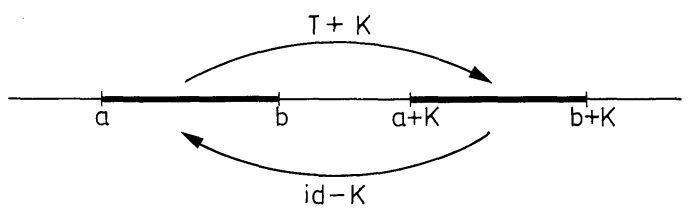

$S=$ 'square root' of $T, T:[a, b]$ D

Fig. 5

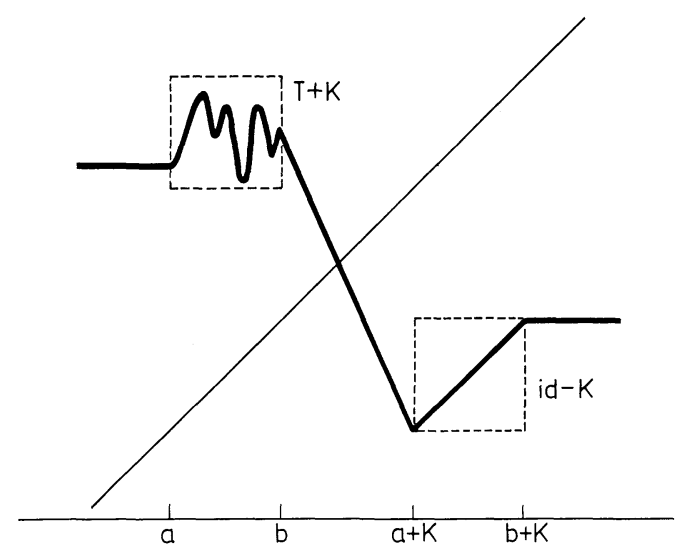

$S=$ 'square root' of $T$

Fig. 6

\section{G. Comments}

(a) Theorem 7 of [1] states that if $a<b$ are two elements of a periodic orbit $\omega$ of period $n$, then between $a$ and $b$ must lie a fixed point of $T^{s}$ for some $s<k$. For let $a=\#\left\{s: 1 \leqq s<k, T^{s} a<a\right\}=\#\{x \in \omega: x<a\}$ and $\beta=\#\left\{s: 1 \leqq s<k, T^{s} b<b\right\}$ $=\#\{x \in \omega: x<b\}$. Clearly $\beta>\alpha \geq 0$ and so there exists $s$ such that $1 \leqq s<k, T^{s} a>a$, and $T^{s} b<b$. For example, the interval labelled 3 in Figure 4 meets an orbit of period 4.

(b) The proof of the main theorem remains valid if we assume that $T$ is a continuous map $X \rightarrow \mathbb{R}$, where $X$ is an arbitrary interval in $\mathbb{R}$.

(c) If $\omega$ is a periodic orbit of $T$ with period $n$, and if $n \vdash k$, where $\vdash$ is the ordering defined on p. 237, then $T$ has an orbit of period $k$ which is contained in $\left[\omega_{\min }, \omega_{\max }\right]$. This follows from (b) on taking $X=\left[\omega_{\min }, \omega_{\max }\right]$.

(d) I took (5) and (6) from the paper [4] of Lie and Yorke in which they prove (7) and study some other consequences of the existence of periodic orbits of period 3.

Šarkovskii gives a direct proof of (8) and does not state or use (5) and (6). The assertion (7) is a special case of his main theorem.

(e) My proof of (12) is different from Šarkovskii's, which seems to break down for $n=3$ and 4 . The proof of (15) is a slight modification of the proof of Sarkovskii, which contains some mistakes. 
(f) Guckenheimer [3] gives a different proof of Theorem 1 assuming that $T$ is smooth, $T:[0,1] \supset, T(0)=T(1)=0$, and the derivative of $T$ vanishes at a single point.

(g) As far as I know, Theorem 2 on minimal orbits (Section E) is not stated anywhere in the literature. Sarkovskii gives in [1] the examples reproduced here in Section $\mathrm{F}(\mathrm{a})$, without pointing out that these examples are essentially the only possible ones. It seems likely though that he was aware of this fact.

\section{H. An Application}

Bowen and Franks prove in [2] the following theorem.

(BF) Let $T:[0,1] \supset$ be continuous and assume that $\operatorname{Per}(T ; n) \neq \emptyset$, where $n=2^{d} m$ and $m \geqq 3$ is odd. Then

(i) the topological entropy $h(T)$ of $T$ is $>\frac{1}{n} \log 2$;

(ii) there exists $K_{n}$ (independent of $T$ ) such that if $r=2^{d} k$ and $k \geqq K_{n}$, then $\# P(T, r) \geqq 2^{r / n}$.

Combining the approach of Bowen and Franks with Šarkovskii's results and with Theorem 2 as indicated below, it is easy to strengthen both of these estimates. We first remark that for $n=3$ a considerably simplified version of the argument of Bowen and Franks yields

(a) $h(T) \geqq \log x, \quad x=\left(1+5^{1 / 2}\right) / 2 \doteq 1.618$.

If now $n$ is odd, then, by Theorem 1 or directly by $(11), \operatorname{Per}\left(T^{2}, 3\right) \neq \emptyset$. Since $h\left(T^{2}\right)$ $=2 h(T)$, this gives

(b) $h(T) \geqq \log x^{1 / 2}$.

Note that, in contrast to (BF(i)), the right-hand side of (b) does not tend to zero when $n=2 k+1$ tends to infinity. From (b) it follows at once that, under the assumptions of $(\mathrm{BF})$,

(c) $h(T) \geqq 2^{-d} \log x^{1 / 2}$.

The estimate (c) can be further improved if we use Theorem 2:

Theorem 3. If $T$ satisfies the assumptions of (BF), then

(i) $h(T)>2^{-d} \log 2^{1 / 2}$;

(ii) there exists a constant $K_{m}$ (independent of $T$ and $d$ ) such that if $r=2^{d} k$ and

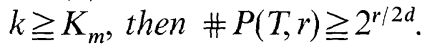

Proof. As in [2], we may assume that $n$ is odd. Taking $n=m$ as small as possible and using Theorem 2, we find that $T$ has a periodic orbit $\omega$ isomorphic to $M_{m}$. Subdivide the interval $\left[\omega_{\min }, \omega_{\max }\right]$ as in Figure 4, replacing the fixed point $S$ if necessary by an interval $S=[\alpha, \beta]$ where $\alpha$ (resp. $\beta$ ) is the smallest (resp. largest) fixed point of $T$ between $\omega^{U}$ and $\omega^{D}$. In view of (9) and the estimate (a) above we may assume that $T$ has no fixed point elsewhere in $\left[\omega_{\min }, \omega_{\max }\right]$.) Labelling the subintervals of 
$\left[\omega_{\min }, \omega_{\max }\right] \backslash S$ as in Figure 4 , we obtain the $m \times m$ incidence matrix

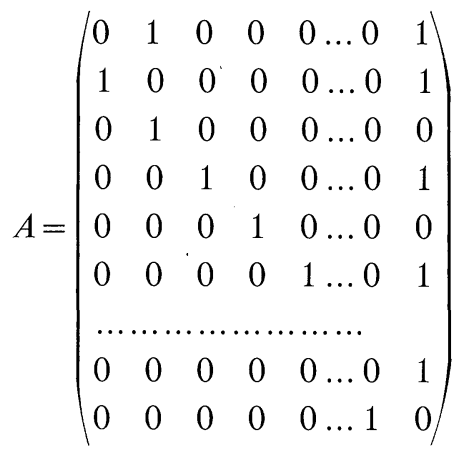

with the characteristic polynomial $-f(\lambda)$, where

(d) $f(\lambda)=\lambda^{m}-2 \lambda^{m-2}-1$.

It is easy to see that $f$ has exactly 3 real roots : $\lambda_{\min },-1$, and $\lambda_{\max }$, and that, for $m \geqq 5$,

(e) $-2^{1 / 2}<\lambda_{\min }<-(2 m /(m+2))^{1 / 2}$ and $2^{1 / 2}<\lambda_{\max }<2^{1 / 2}+2^{-(m+3) / 2}$.

Using the argument of Bowen and Franks, it is sufficient to show that, for large $r$

(f) $\operatorname{Trace} A^{r} \sim \lambda_{\text {max }}^{r}$.

As in [2], it can be shown that (f) follows from the Perron-Frobenius theorem. We can, however, obtain a more precise information directly: we claim that

(g) $\left|\lambda_{i}\right|<1$ for $1 \leqq i \leqq m-3$,

where $\lambda_{i}$ are the remaining $m-3$ roots of $f$. A simple proof of $(\mathrm{g})$, for which I am indebted to A. Connes, goes as follows. Set $g=\lambda^{m}-2 \lambda^{m-2}$ and note that, for every sufficiently small $\varepsilon>0$,

$$
1=|f-g|<|g| \text { on the circle }|\lambda|=1+\varepsilon .
$$

Hence, by Rouché's theorem, $f$ has exactly $m-2$ zeros in the disc $|\lambda| \leqq 1$. It is easy to check that $\lambda=-1$ is the only root of $f$ on the unit circle, proving $(\mathrm{g})$ and so (in combination with the arguments in [2]) concluding the proof of Theorem 3.

We note that (g) can be used, together with (e) and the inequalities on pp. 7 and 8 of [2], to obtain an explicit upper bound for the constant $K_{m}$.

\section{References}

1. Šarkovskii, A.N. : Coexistence of cycles of a continuous map of a line into itself. Ukr. Mat. Z. 16, 6171 (1964)

2. Bowen, R., Franks,J.: The periodic points of maps of the disk and the interval. I.H.E.S. preprint, November 1975

3. Guckenheimer,J.: On the bifurcation of maps of the interval. Preprint, 1976

4. Li,T., Yorke,J. A.: Period three implies chaos. Am. Mat. Monthly 82, 985-992 (1975)

Communicated by J. L. Lebowitz

Received February 9, 1977 